\title{
RTEC-intrinsic IL-17-driven inflammatory circuit amplifies antibody-induced glomerulonephritis and is constrained by Regnase-1
}

\author{
De-Dong Li, ${ }^{1}$ Rami Bechara, ${ }^{1}$ Kritika Ramani, ${ }^{1}$ Chetan V. Jawale, ${ }^{1}$ Yang Li, ${ }^{1}$ Jay K. Kolls, ${ }^{2}$ \\ Sarah L. Gaffen, ${ }^{1}$ and Partha S. Biswas ${ }^{1}$ \\ ${ }^{1}$ University of Pittsburgh, Division of Rheumatology and Clinical Immunology, Pittsburgh, Pennsylvania. USA. ${ }^{2}$ Tulane \\ University, Department of Medicine, New Orleans, Louisiana, USA.
}

\begin{abstract}
Antibody-mediated glomerulonephritis (ACN) is a clinical manifestation of many autoimmune kidney diseases for which few effective treatments exist. Chronic inflammatory circuits in renal glomerular and tubular cells lead to tissue damage in ACN. These cells are targeted by the cytokine IL-17, which has recently been shown to be a central driver of the pathogenesis of AGN. However, surprisingly little is known about the regulation of pathogenic IL-17 signaling in the kidney. Here, using a well-characterized mouse model of AGN, we show that IL-17 signaling in renal tubular epithelial cells (RTECs) is necessary for ACN development. We also show that Regnase-1, an RNA binding protein with endoribonuclease activity, is a negative regulator of IL-17 signaling in RTECs. Accordingly, mice with a selective Regnase-1 deficiency in RTECs exhibited exacerbated kidney dysfunction in AGN. Mechanistically, Regnase-1 inhibits IL-17-driven expression of the transcription factor $I_{\kappa} B \xi$ and, consequently, its downstream gene targets, including $/ / 6$ and $L c n 2$. Moreover, deletion of Regnase- 1 in human RTECs reduced inflammatory gene expression in a IКB $\xi$-dependent manner. Overall, these data identify an IL-17-driven inflammatory circuit in RTECs during AGN that is constrained by Regnase-1.
\end{abstract}

Conflict of interest: The authors have declared that no conflict of interest exists.

Copyright: () 2021, Li et al. This is an open access article published under the terms of the Creative Commons Attribution 4.0 International License.

Submitted: January 11, 2021

Accepted: May 19, 2021

Published: July 8, 202

Reference information: JCI Insight. 2021;6(13):e147505.

https://doi.org/10.1172/jici. insight.147505.

\section{Introduction}

Antibody-mediated glomerulonephritis (AGN) describes a heterogenous group of renal conditions caused by an inappropriate response to renal autoantigens, such as the glomerular basement membrane (GBM) $(1,2)$. The most severe form of human AGN is crescentic glomerulonephritis $(G N)$, characterized by the formation of glomerular crescents and tubulointerstitial inflammation (3, 4). Although the initiators of AGN differ among diseases, the terminal events in end-organ kidney damage share common mechanisms that remain poorly understood. Even with the most aggressive immunosuppressive regimens, as many as approximately $30 \%$ of nephritic patients develop end-stage renal disease (5). To date, the best therapies are broad-acting immunosuppressants, namely cyclophosphamide with corticosteroids (5). Gaining a fundamental understanding of how pathogenic kidney inflammation is driven and amplified could help fill an unmet clinical need to achieve more specific and effective responses in AGN.

Typically, immune-mediated tissue pathology is viewed through the lens of hematopoietic lymphoid and myeloid cells. Less appreciated is the contribution of organ-specific tissue cells as amplifiers of inflammation that ultimately lead to chronic disease. In the kidney, glomerular and tubular inflammation are hallmark features of AGN (6). In fact, the majority of AGN patients progress to end-stage renal disease as a consequence of chronic tubular inflammation (7). Renal tubular epithelial cells (RTECs) comprise the major cell type of the tubular compartment (8). These cells actively contribute to the production of inflammatory mediators that propagate tissue injury locally by secreting cytokines and chemokines and undergoing apoptosis (7). Even so, our understanding of the inflammatory circuits operative in RTECs during chronic kidney disease is surprisingly rudimentary.

AGN has traditionally been considered a B cell-dependent disease because of the high autoantibody levels found in these patients (9). However, multiple studies also implicate IL-17, the signature cytokine of Th17 cells, as an essential driver of AGN pathology (10-15). IL-17 fuels an inflammatory response that 
damages renal podocytes, causing proteinuria. Loss of glomerular filtration is followed by IL-17-dominated tubular inflammation and damage of RTECs. Interestingly, IL-17 signaling in the nephritic kidney has no impact on the immunocomplex deposition in the glomeruli (10). While IL-17 is secreted almost exclusively by lymphocytes, its target cells are often but not always nonhematopoietic $(16,17)$. The specific IL-17 target cells in the context of renal autoimmunity remain unclear.

Although not well defined in the kidney, dysregulation of Th17 cells or IL-17 signaling causes pathology in other autoimmune conditions, such as psoriasis and psoriatic arthritis, where anti-IL-17 biologics are now standard therapies $(18,19)$. A landmark recent study showed that human Th17 cells specific for commensal microbes such as Staphylococcus aureus and Candida albicans contribute to AGN (20). These findings are consistent with prior work in preclinical models that IL-17 and its adaptor Act1 promote AGN in mice $(10-14,21-23)$. The downstream events operative in AGN have been presumed to occur through IL-17-mediated activation of the NF- $\mathrm{kB}$ signaling pathway, which is induced in response to a myriad of immune stimuli, including IL-17, TNF- $\alpha$, TLR ligands, and T cell signaling $(24,25)$. NF- $\kappa$ B induces a panoply of genes that promote renal inflammation, including IL-6, neutrophil-attracting chemokines (CXCL1, CXCL5), and lipocalin 2 (LCN2, NGAL, 24p3), a major biomarker and driver of renal inflammation

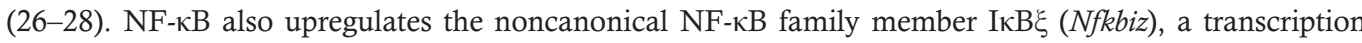
factor that regulates a more restricted subset of genes that includes both IL-6 and LCN2 $(29,30)$. In recent years, it has become evident that IL-17 regulates many genes posttranscriptionally, either by controlling mRNA stabilization and/or by influencing translation of downstream transcripts (31-33). Among these posttranscriptionally regulated targets are $I l 6$ and $N f k b i z(\mathrm{I} \kappa \mathrm{B} \xi)$.

Autoimmune signaling must be sufficiently restrained in order to prevent hyperactivation of pathways that could contribute to immunomediated pathology. The endoribonuclease Regnase-1 (MCPIP1, encoded by $Z c 3 h 12 a$ ) is best recognized as an inhibitor of TLR and TCR signaling (34). In CD4 ${ }^{+} \mathrm{T}$ cells, loss of Regnase-1 leads to markedly elevated IL-6 levels and, consequently, elevated Th17 differentiation and exacerbated autoimmune disease, demonstrated in the experimental autoimmune encephalitis (EAE) model of multiple sclerosis (35). In cultured fibroblasts, Regnase-1 has been shown to restrict IL-17 signaling by degrading downstream target mRNAs such as Il6 (33). With respect to kidney, however, little is known, though a global deficiency of Regnase- 1 enhances the immune response to disseminated $C$. albicans, a fungal infection that targets the kidney (33).

In this report, we show that RTECs are critical mediators of inflammatory damage in AGN by virtue of their sensitivity to IL-17 signaling. Conversely, Regnase-1 restricts AGN pathogenesis by limiting IL-17-driven renal-specific expression of the transcription factor I $\mathrm{KB} \xi$ and, consequently, its downstream gene targets $I l 6$ and Lcn2 within RTECs. These findings demonstrate a potent, kidney-intrinsic inflammation circuit that is potentiated by RTEC-specific IL-17 signaling and restrained by Regnase-1 in a highly cell type-specific manner.

\section{Results}

RTEC-intrinsic IL-17 signaling is critical for AGN pathology. AGN is mediated by both kidney-infiltrating immune cells (hematopoietic) and kidney-resident nonhematopoietic cells. Several studies show that IL-17 and its receptor (IL-17RA), through the adaptor Act1, are essential for AGN (10-14, 21). However, the identity of IL-17 target cells in this setting is unknown. To define the contribution of IL-17RA in hematopoietic versus nonhematopoietic cells, we created BM chimeric mice in which $1117 \mathrm{ra}^{-1-}$ or WT BM was adoptively transferred into irradiated reciprocal hosts. Successfully reconstituted recipients were then evaluated for susceptibility to AGN by injecting mice with rabbit IgG in complete Freund's adjuvant (CFA), followed 3 days later by injection with rabbit anti-GBM serum $(10,36)$. As shown, WT hosts receiving $1117 \mathrm{ra}^{-/-} \mathrm{BM}$ showed identical susceptibility to AGN as WT mice, indicating that any activity of IL-17 in hematopoietic cells is dispensable for AGN pathology. Conversely, $I l 17 \mathrm{ra}^{-/-}$mice, regardless of BM source, were resistant to AGN (Figure 1A). Thus, IL-17RA signaling in nonhematopoietic cells is required for development of AGN.

The kidney-intrinsic nonhematopoietic cells thought to mediate AGN are RTECs. Prior studies showed that IL-17 can act on primary cultured RTECs to induce expression of cytokine and chemokine genes that are known to drive inflammation in AGN (24). Based on this, we sought to define the role of IL-17 signaling in RTECs in vivo. We took advantage of well-characterized Cdh16 ${ }^{\text {Cre }}$ mice, in which the Cre recombinase is expressed in the epithelial lining of proximal tubules, collecting ducts, loops of Henle, and distal tubules (37). We crossed $I l 17 \mathrm{ra}^{f l f l}$ mice to Cdh16 ${ }^{\text {Cre }}$ mice (termed $I l 17 \mathrm{ra}^{\text {Cdh10}}$ ), which selectively and efficiently depleted IL-17RA in RTECs, as previously reported (38). Following 
A

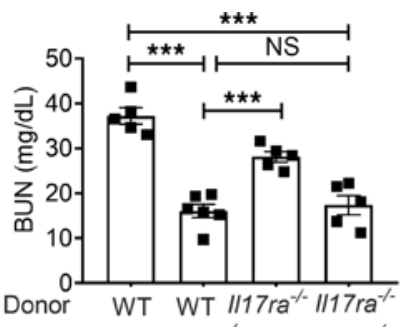

Recipient WT $\| 17 \mathrm{ra}^{\%}$ WT $\| 17 \mathrm{ra}^{\%}$
B
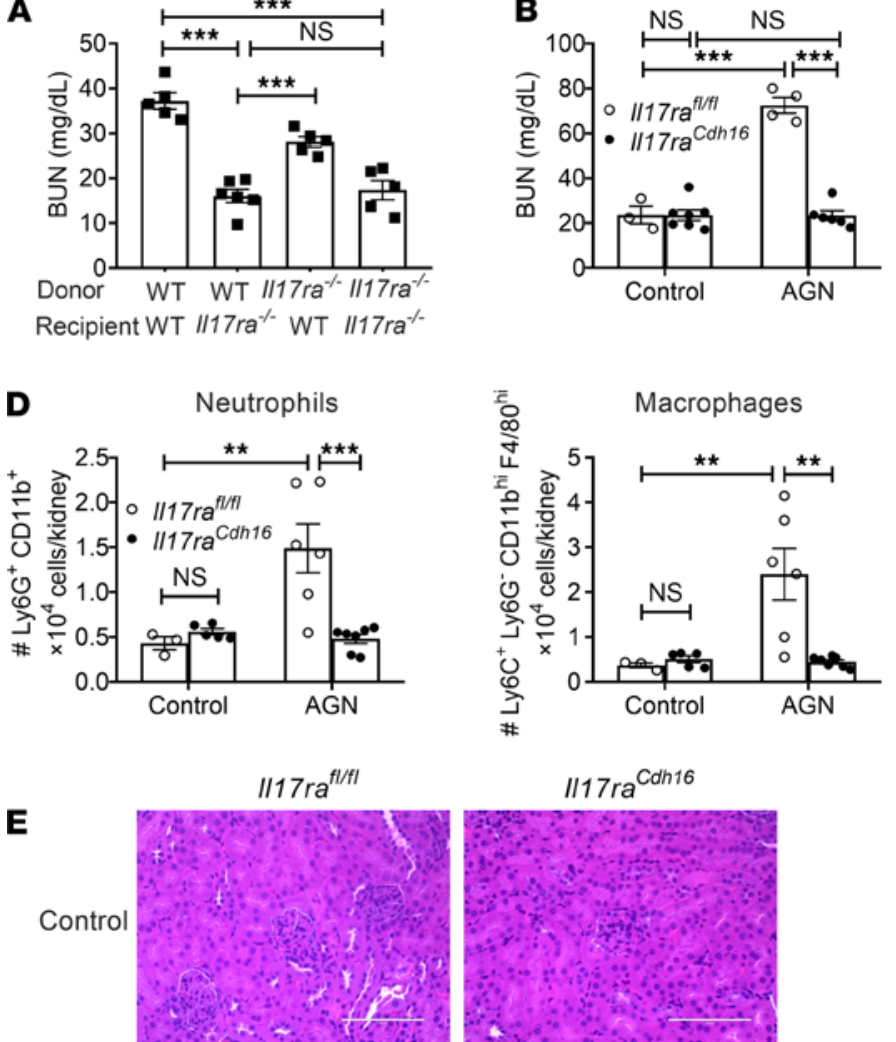

D

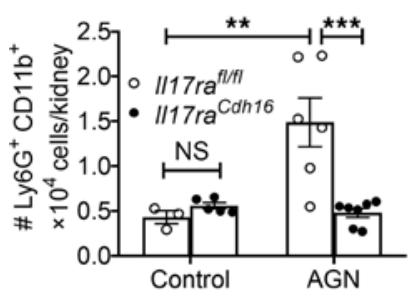

E

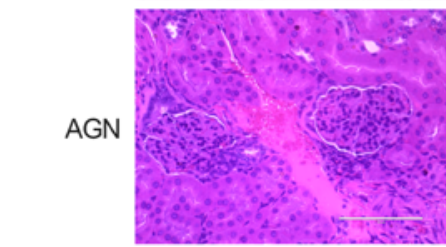

$\| 117 r^{f / / f t}$

$\mathbf{F}$

Control

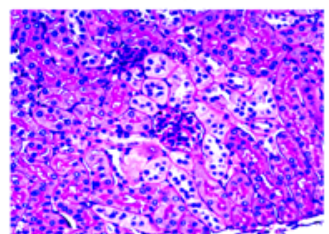

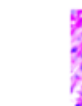

AGN

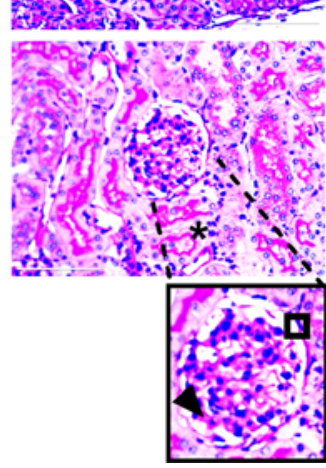

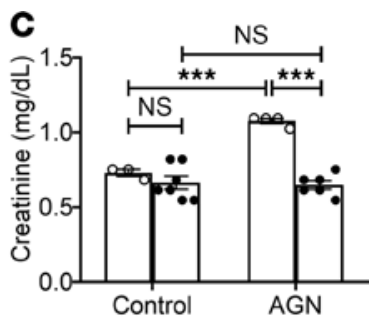
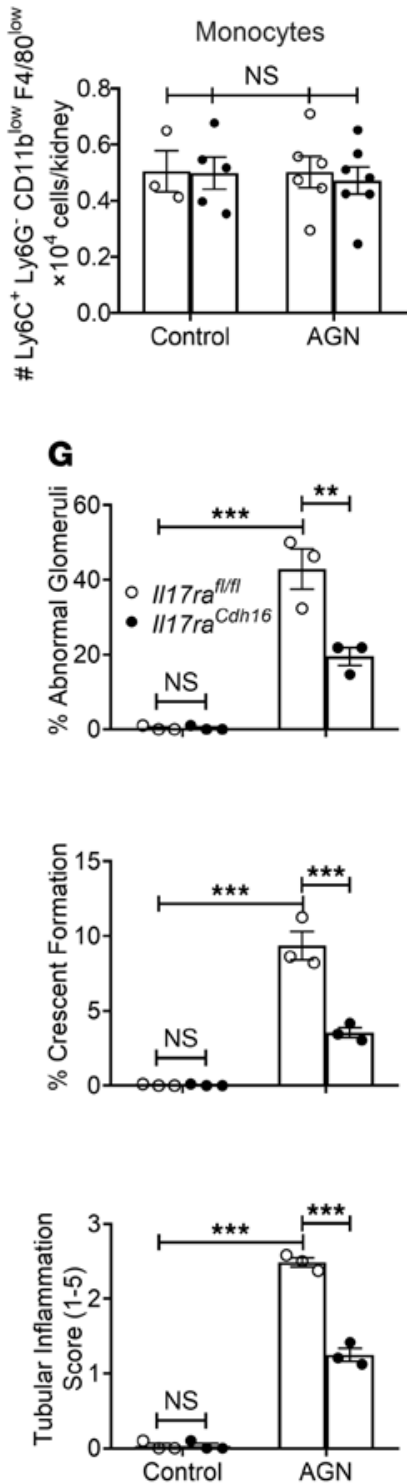

Figure 1. IL-17RA signaling in RTECs is required for AGN. (A) BM cells from I/17ra ${ }^{-1-}$ (CD45.2+) and WT (CD45.1 $1^{+}$) mice were adoptively transferred into sublethally irradiated $I 117 \mathrm{ra}^{-1-}$ or WT recipients $(n=5-6)$. Eight weeks later, successfully reconstituted mice were subjected to AGN and assessed for kidney dysfunction by measuring serum BUN levels. I117 $\mathrm{ra}^{f / / f l}$ and $/ 117 \mathrm{ra}^{\text {Chh16 }}$ mice $(n=3-7)$ were subjected to AGN. (B and C) At day 14 after anti-GBM serum injection, serum BUN (B) and serum creatinine (C) levels were measured by ELISA. (D) Neutrophil, macrophage, and monocyte infiltration in the kidney was quantified by flow cytometry at day 7 p.i. (E and F) Representative photographs of H\&E-stained (E) and PAS-stained (F) renal histopathology were assessed. (C) Renal pathology was blindly evaluated and scored for percentages of abnormal glomeruli and crescent formation and for tubular inflammation. Data representative of 1 of 3 mice/group for $\mathbf{E}$ and $\mathbf{F}$. A small part (as indicated by dotted lines) of the original image (total original magnification, $\times 400$ ) was shown as inset panels. Open square, indicating entire glomerulus with mesangial and endocapillary hypercellularity; black arrow, GBM thickening; asterisk, tubular atrophy. Data pooled from at least 2 independent experiments. Statistical analysis by 1-way ANOVA (A) and 2-way ANOVA (B-D, G). ${ }^{* *} P<0.01 ;{ }^{* *} P<0.001$. 
AGN, Ill7ra ${ }^{\text {Cdh16 }}$ mice showed diminished levels of serum BUN and creatinine (Figure 1, B and C). Il17ra ${ }^{\text {Cdh16 }}$ kidneys exhibited compromised renal infiltration of neutrophils and macrophages at day 7 after AGN (Figure 1D and Supplemental Figure 1; supplemental material available online with this article; https://doi.org/10.1172/jci.insight.147505DS1). Interestingly, the number of inflammatory monocytes, $\mathrm{CD}^{+} \mathrm{T}, \mathrm{CD} 8^{+} \mathrm{T}$, and B cells were comparable between the groups (Figure 1D, Supplemental Figures 1, and Supplemental Figure 2, A and B). When evaluated for kidney pathology, $I 117 \mathrm{ra}^{\text {Cdh16 }}$ kidneys showed reduced mesangial and endocapillary hypercellularity, thickening of GBM, crescents formation, tubular atrophy, and tubular inflammatory cell infiltration in comparison with controls, similar to $I l 17 \mathrm{ra}^{-/-}$or Act1 $^{-1-}$ mice $(10,14)$ (Figure 1, E-G). These results, thus, demonstrate an RTEC-specific role of IL-17R signaling in AGN.

Regnase-1 haploinsufficient mice show exaggerated $A G N$. Surprisingly little is known about the molecular signals that constrain inflammation in the context of AGN. Regnase-1 (encoded by Zc3h12a) is a negative feedback regulator of many inflammatory stimuli, including the TCR, TLR4, and IL-17 (33, 34). Mice lacking Zc3h12a entirely show systemic inflammation and early mortality, an effect that we previously found to be independent of IL-17 $(39,40)$. In contrast, $Z c 3 h 12 a$ haploinsufficient $\left(Z c 3 h 12 a^{+/-}\right)$mice have no peripheral inflammation in kidney or other visceral organs but exhibit enhanced susceptibility to IL-17-induced pathology in certain settings, including EAE $(33,41,42)$. Consequently, we subjected $Z c 3 h 12 a^{+/-}$mice to AGN to determine if this molecule influences outcomes in renal autoimmunity. As shown, $Z c 3 h 12 a^{+/-}$mice were markedly more susceptible to AGN than control mice (Figure 2A). Mice lacking a single copy of the $Z c 3 h 12 a$ gene demonstrated increased infiltration of neutrophils and macrophages but not monocytes, $\mathrm{CD} 4^{+} \mathrm{T}, \mathrm{CD} 8^{+} \mathrm{T}$, and $\mathrm{B}$ cells (Figure 2B, Supplemental Figure 1, and Supplemental Figure 3, A-C). Moreover, kidney dysfunction was reversed in $Z c 3 h 12 a^{+/-} I l 17 \mathrm{ra}^{-/-}$mice, indicating that IL-17 signaling is essential for Regnase-1-mediated inhibitory signaling in the nephritic kidney (Figure 2C).

Regnase-1 can also restrain Th17 cell differentiation; hence, mice lacking Regnase- 1 in $\mathrm{CD}^{+} \mathrm{T}$ cells have exacerbated disease in EAE (35). Thus, the increased nephritis in $Z c 3 h 12 a^{+/-}$could be due to enhanced IL-17 production from T cells or to unrestrained IL-17 signaling in responder cells - or potentially both. To distinguish among these possibilities, we created BM chimeric mice where $Z c 3 h 12 a^{+/-}$or $Z c 3 h 12 a^{+/+} \mathrm{BM}$ from littermates were adoptively transferred into irradiated reciprocal hosts. Successfully reconstituted mice were subjected to AGN. These data show that Regnase-1 in nonhematopoietic cells was required for AGN development, evidenced by increased serum BUN and creatinine (Figure 2D and Supplemental Figure 3D). $Z c 3 h 12 a^{+/-}$animals receiving either WT or $Z c 3 h 12 a^{+/-}$BM showed evidence of increased glomerular pathology, as characterized by exaggerated crescents formation, mesangial hypercellularity, and increased GBM thickness. Additionally, these animals exhibited increased tubular atrophy and tubular inflammatory cell infiltration compared with WT recipients (Figure 2, E-G). These data indicate that Regnase- 1 restricts AGN pathogenesis by acting in nonhematopoietic cells, with the caveat that mice still retained 1 intact copy of the $Z c 3 h 12 a$ gene.

Regnase- 1 restrains AGN severity in an RTEC-intrinsic manner. Given that Regnase-1 restricts IL-17 signaling and that Regnase-1 and IL-17RA both act in nonhematopoietic cells in AGN in an opposing manner, we predicted that loss of Regnase-1 in RTECs would exacerbate tissue pathology in AGN.

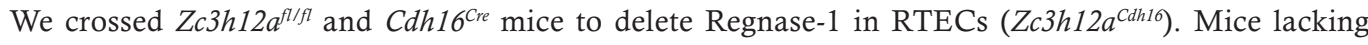
Regnase-1 in RTECs breed normally and displayed normal serum BUN and kidney tissue architecture at baseline (Figure 3, A and D). After AGN induction, compared with Zc3h12a $a^{f l f l}$ controls, Zc3h12a $a^{\text {cdh } 16}$ mice showed more severe kidney dysfunction and a trend toward increased expression of the classical kidney injury marker Kim1 (43) (Figure 3, A-C). Moreover, Zc3h12a ${ }^{\text {Cdh16 }}$ mice exhibited more glomerular abnormality as characterized by increased mesangial and endocapillary hypercellularity, thickening of GBM, and crescents formation following AGN (Figure 3, D-F). Zc3h12a cdh16 kidneys demonstrated exaggerated tubular atrophy and inflammatory cell infiltration in the tubular space compared with control mice. $Z c 3 h 12 a^{C d h 16}$ kidney also showed increased expression of multiple chemokines implicated in AGN, including $C x c l 1, C x c l 2, C c l 20$, and $C c l 2$, that are known to recruit immune cells to diseased

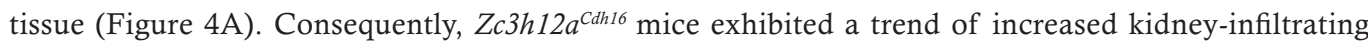
$\mathrm{CD} 45^{+}$inflammatory cells (Supplemental Figure 1 and Supplemental Figure 4A). Although the number of neutrophils and macrophages were increased in $Z c 3 h 12 a^{C d h 16}$ mice, the frequencies of monocytes, $\mathrm{CD}^{+} \mathrm{T}, \mathrm{CD} 8^{+} \mathrm{T}$, and $\mathrm{B}$ cells were comparable among the nephritic groups (Figure $4, \mathrm{~B}$ and $\mathrm{C}$, 
A

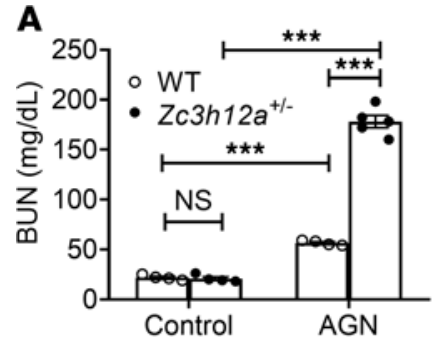

c

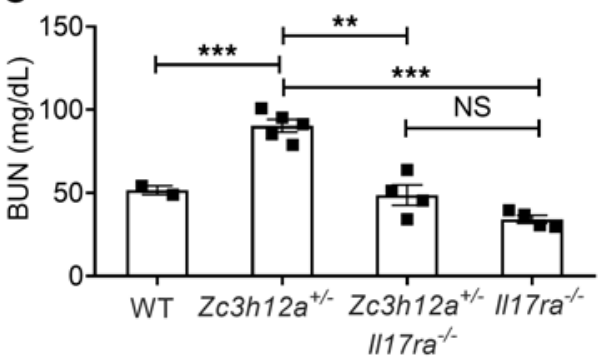

E
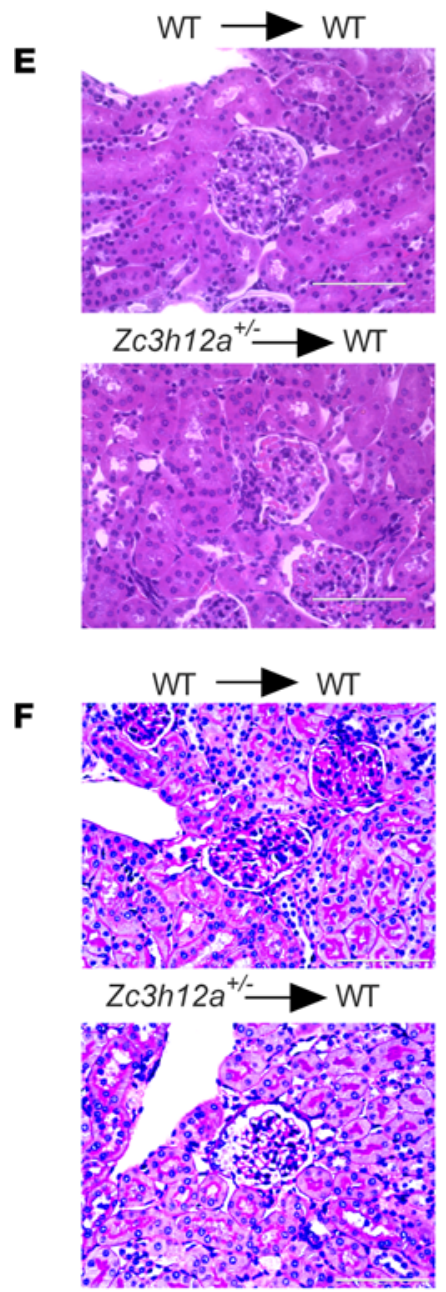
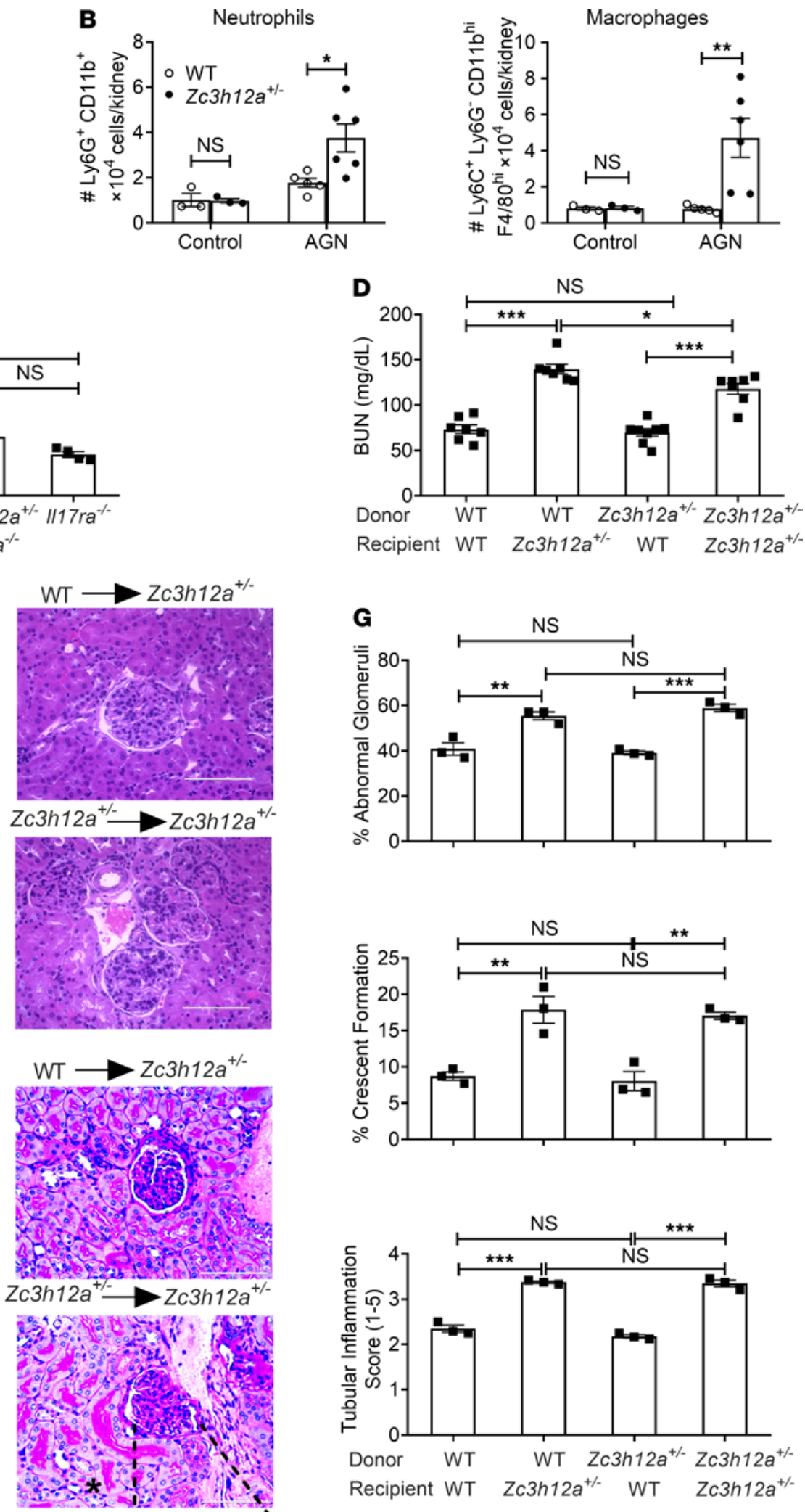
Figure 2. Zc3h12a $a^{+-}$mice show exaggerated AGN. (A) Zc3h12a $a^{+/}(\mathrm{WT})$ and $Z c 3 h 12 a^{+/}(n=4-5)$ were subjected to AGN. Mice were evaluated for kidney dysfunction by measuring serum BUN at day 14 p.i. (B) Neutrophil and macrophage infiltration in the kidney was quantified by flow cytometry at day 7 p.i. (C) WT,

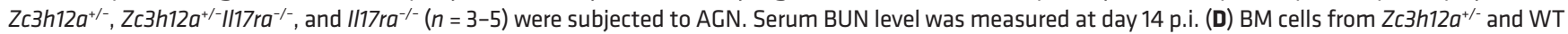
mice were adoptively transferred into sublethally irradiated $Z c 3 h 12 a^{+/-}$or WT recipients $(n=5-7)$. Eight weeks later, successfully reconstituted mice were subjected to AGN and assessed for serum BUN levels. (E and F) Representative photographs of H\&E-stained (E) and PAS-stained (F) renal histopathology. Data representative 1 of 3 mice/group. A small part (as indicated by dotted lines) of the original image (total original magnification, $\times 400$ ) was shown as inset panels. Open square, indicating entire glomerulus with mesangial and endocapillary hypercellularity; black arrow, GBM thickening; asterisk, tubular atrophy. (C) Renal pathology was blindly evaluated and scored for percentages of abnormal glomeruli and crescent formation, and for tubular inflammation. Data pooled from at least 2 independent experiments. Statistical analysis by 2 -way ANOVA (A and $\mathbf{B}$ ) and 1-way ANOVA (C, D, and $\mathbf{G}) .{ }^{*} P<0.05 ;{ }^{* *} P<0.01 ;{ }^{* *} P<0.001$.

Supplemental Figure 1, and Supplemental Figure 4B). These results indicate a role for Regnase-1 as a negative regulator of renal inflammation.

Regnase- 1 functions by binding to and degrading client mRNA transcripts (33). Some inflammatory transcripts are direct targets of Regnase-1, such as Il6, where Regnase-1 binds to a stem-loop sequence in $I l 6$ the $3^{\prime}$ UTR to promote degradation. However, many mRNAs are indirectly influenced by Regnase- 1 through its capacity to degrade mRNA, including the gene encoding the I $\kappa \mathrm{B} \xi$ transcription factor (Nfkbiz). A major example of IкB $\xi$-dependent mRNA is $L c n 2$ (44). Therefore, we examined expression of known Regnase-1 client transcripts involved in AGN pathology in Zc3h12a cdhl6 mice. There was no difference in the expression of Il6, Nfkbiz, or Lcn2 in kidney at baseline (Figure 4, D-F). However, following AGN, there was a marked increase in levels of Il6, Nfkbiz, and Lcn2 in Zc3h12a cahlo kidneys compared with controls. Notably, all these genes are also known targets of IL-17 in AGN and are reduced in $I 117 \mathrm{ra}^{-/-}$mice subjected to AGN (10). Collectively, these data suggest a role for Regnase-1 in restraining expression of pathogenic IL-17-dependent genes in an RTEC-specific manner during the inflammatory process of AGN.

Regnase-1 in human RTECs drives inflammation via I $k b \xi$. To determine in a more direct way how Regnase-1 functions in RTECs, we deleted the ZC3H12A gene in the human tubular kidney 2 (HK-2) cell

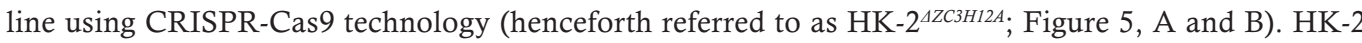
is a tubular epithelial cell line derived from normal kidney, and we found it to be highly responsive to IL-17 (Figure 5C). HK-2 2 IZC3HI2A and control HK-2 cells were stimulated with IL-17 and assessed for mRNA and protein expression of known IL-17 responsive genes, including $I L 6, L C N 2$, NFKBIZ, CEB$P B$, and $C E B P D$. We also performed stimulations in conjunction with TNF- $\alpha$, since IL-17 and TNF- $\alpha$ often signal in a potently synergistic manner (45). IL-17 alone or in conjunction with TNF- $\alpha$ upregulated transcript (Figure 5, C and E) and protein (Figure 5, D and F) expression of $I L 6$ and LCN2 in HK-2 ${ }^{4 Z}$ ${ }^{C 3 H 12 A}$ cells. Notably, HK-2 ${ }^{Z C 3 H 12 A}$ cells demonstrated an increased expression of NFKBIZ transcript and Iкb $\xi$ expression following IL-17 treatment (Figure 5, G and H). The impact of IL-17 alone or IL-17 plus TNF- $\alpha$ on the mRNA and protein expression of $C E B P B$ and $C E B P D$ was variable (Supplemental Figure 5, A and B). Additionally, a luciferase assay revealed activation of $L c n 2$ promoter activity following IL-17 stimulation in human embryonic kidney 293 cells (HEK-293) (Supplemental Figure 5C). Overall, these results indicate that Regnase-1 is a negative regulator of IL-17 signaling in human RTECs.

Since Regnase-1 restricts expression of the I $\kappa B \xi$ transcription factor (NFKBIZ), which mediates expression of many of these characteristic downstream mRNAs, we evaluated the impact of IкB $\xi$ in HK-2 cells with respect to IL-17 signaling. To that end, we knocked down NFKBIZ by siRNA in HK-2 ${ }^{2 Z C 3 H 12 A}$ and control HK-2 cells, which resulted in 50\%-60\% reduction in NFKBIZ expression (Figure 6A). HK-2 2 AZC3HI2A cells in which NFKBIZ was silenced showed diminished levels of LCN2 and IL6 mRNA compared with controls (Figure 6B). Interestingly, while CCL20 levels were reduced upon NFKBIZ silencing, CXCL1 and CXCL2 were increased (Figure 6C). These results indicate that Regnase-1 selectively suppresses some IL-17 responsive genes that mediate kidney inflammation by increasing NFKBIZ in human RTECs.

\section{Discussion}

Development of improved therapeutic approaches to treat AGN will require a more comprehensive understanding of the fundamental immune mechanisms in the kidney that drive this disease. The kidney is an immunologically distinct organ and is exquisitely sensitive to end-organ damage in numerous autoimmune diseases and infections. Indeed, renal injury caused by inappropriate immune responses is the most important predictor of mortality in AGN patients (7). Even so, our understanding of the 
A

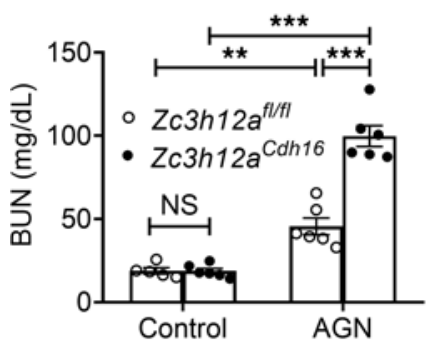

D
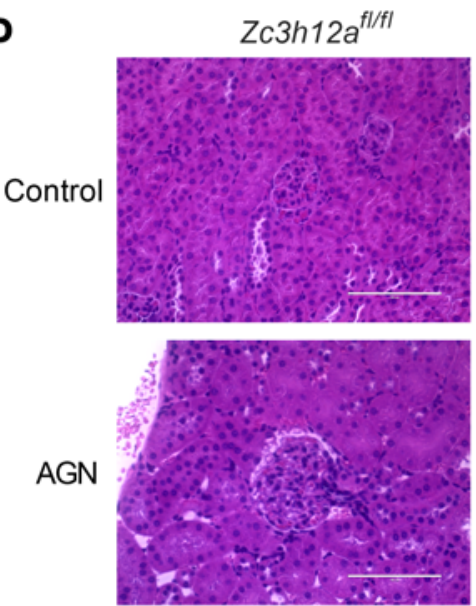

E

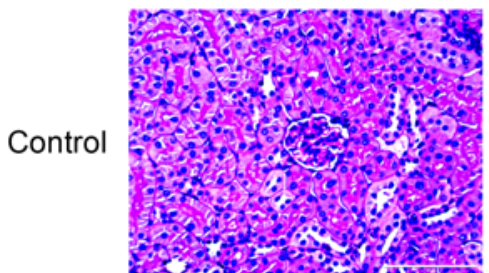

Zc3h12a $a^{f l / f \mid}$

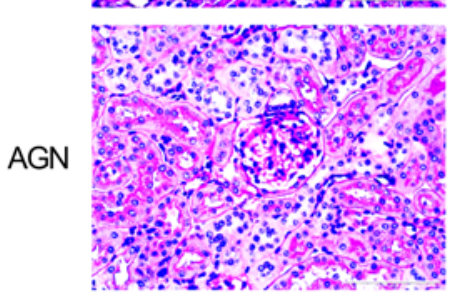

B
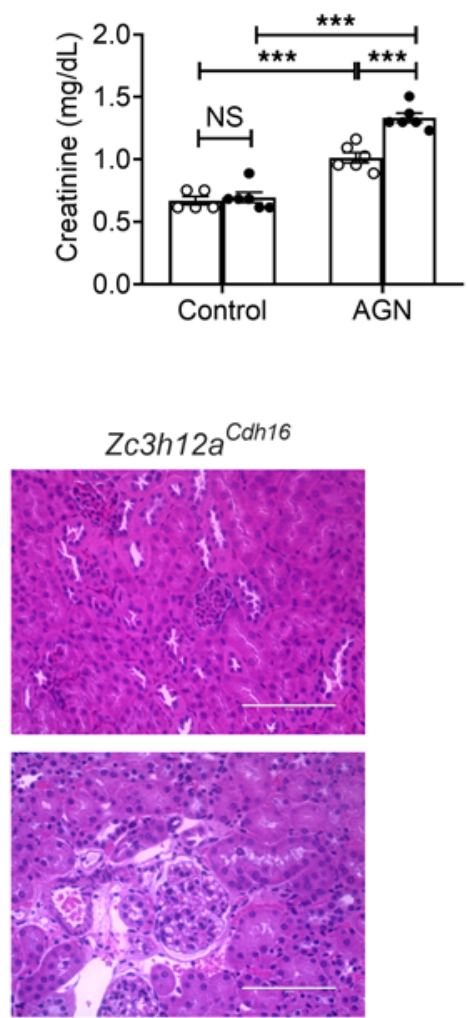

Zc3h12a $a^{C d h 16}$
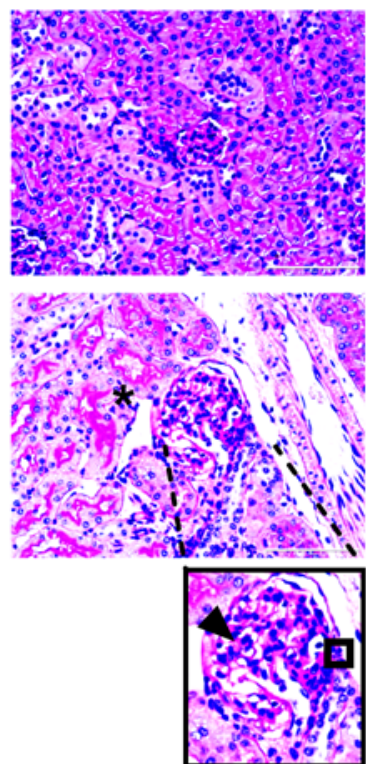

C

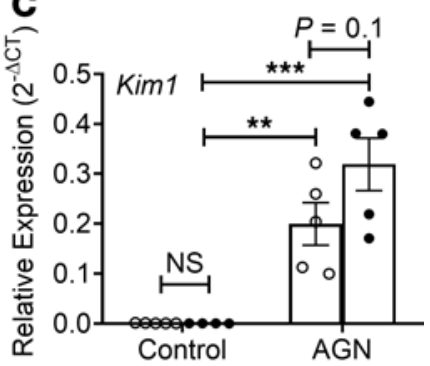

F
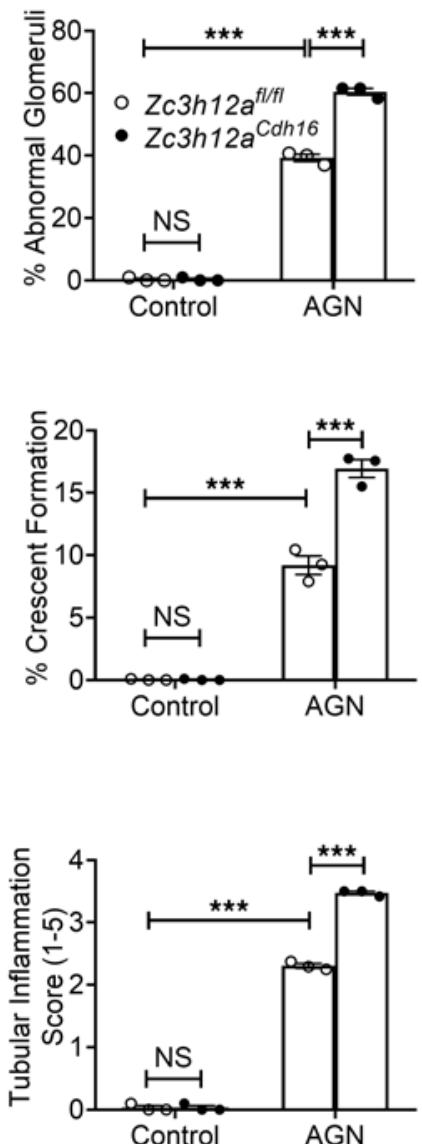

Figure 3. RTEC-specific Regnase-1 deficiency exacerbates AGN. Zc3h12afl/fl and Zc3h12a $a^{\text {chh16 }}$ mice $(n=5-6)$ were subjected to AGN. (A and B) At day 14 p.i., serum BUN (A) and serum creatinine (B) levels were assessed. (C) Kim1 transcript expression was assessed at day 7 p.i. Expression was normalized to Gapdh. (D and E) Representative photographs of H\&E-stained (D) and PAS-stained (E) renal histopathology. (F) Renal pathology was blindly evaluated and scored for percentages of abnormal glomeruli and crescent formation and for tubular inflammation. Data representative of 1 of 3 mice/group for $\mathbf{D}$ and E. A small part (as indicated by dotted lines) of the original image (total original magnification, $\times 400$ ) was shown as inset panels. Open square, indicating entire glomerulus with mesangial and endocapillary hypercellularity; black arrow, GBM thickening; asterisk, tubular atrophy. Data pooled from at least 2 independent experiments. Statistical analysis by 2 -way ANOVA. ${ }^{* *} P<0.01 ;{ }^{* *} P<0.001$. 
fundamental immune processes in the kidney lags considerably behind that of other visceral organs, such as the gut or liver $(1,46)$. This is due to multiple kidney-specific factors, including poor regenerative capacity of nephrons, uremic toxins, hypoxia, and arterial blood pressure, which have confounding impacts on the ongoing immune response in the kidney. Defining how IL-17 mediates signals in target cell types in the nephritic kidney has important therapeutic implications, as specific targeting of the IL-17 pathway in these kidney-resident cells could spare other organs from unwanted side effects and potentially preserve important IL-17 host-defensive activities.

IL-17 is a critical contributor of autoinflammatory diseases (47). Antibodies that neutralize IL-17 were approved in 2016 and show efficacy in psoriasis, psoriatic arthritis, and ankylosing spondylitis $(18,19)$. In contrast, IL-17 blockade caused adverse outcomes in Crohn's disease due to an unexpectedly strong tissue-reparative effect of intestinal epithelium (48-50). These dichotomous outcomes highlight the ramifications of tissue-specific mechanisms of IL-17 signaling. While there has been much research effort describing IL-17-producing cells and their generation, far less is known about how IL-17 mediates downstream signal transduction and mechanisms that regulate this process in responding cells. We showed that RTEC-specific activity of the IL-17 receptor subunit IL-17RA drives chronic inflammatory response in the nephritic kidney. Notably, IL-17RA acts as a shared receptor subunit for the related cytokines IL-17F and IL-17C (51). Hence, some of the effects seen here could be attributable to these cytokines, as well. Despite their emerging role in AGN, both IL-17F and IL-17C signaling mechanisms in the RTECs are almost entirely undefined, and no studies to date have focused on the regulation of IL-17F- and IL-17C-dependent signal transduction in these kidney-resident cell types $(52,53)$. Moreover, the type 2 cytokine IL-25 also signals via IL-17RA (51). To date, there is no evidence suggesting a pathogenic role of IL-25 in AGN development. The findings in this study, thus, set the stage for pursuing analysis of these related but poorly understood IL-17 family of cytokines.

The contribution of kidney-resident cells such as RTECs is often overlooked when considering the development of new therapeutic strategies in inflammatory kidney diseases. Although the IL-17R is ubiquitously expressed (51) and some studies indicated that IL-17 can act on immune cells including T cells, B cells, and NK cells $(16,17,54)$, we show that the contribution of the hematopoietic system in AGN is, in fact, negligible. Rather, IL-17, produced locally in the nephritic kidney by Th17 and $\gamma \delta \mathrm{T}$ cells, signals directly on RTECs to drive production of pathogenic factors including cytokines, chemokines, and LCN2 (10). This finding is in line with other organ-specific autoimmune conditions, including collagen-induced arthritis and imiquimod-induced (IMQ-induced) dermatitis, where a tissue-dependent role for IL-17 signaling was similarly demonstrated. A limitation of BM chimeric mouse studies is that kidney harbors a radio-resistant tissue-resident population of macrophage $\left(\mathrm{CX} 3 \mathrm{CR} 1^{+}\right)$, which are responsive to IL-17 (55). Hence, future studies will need to address the role of pathogenic IL-17 signaling in tissue-resident macrophages in AGN (e.g., by crossing $C x 3 c r 1^{C r e}$ mice to $I l 17 \mathrm{ra}{ }^{f l / f l}$ animals).

Regnase-1 has been shown to restrict many inflammatory signals, and IL-17 is just one of many. Based on our studies, we cannot rule out the roles for Regnase- 1 in restricting other tissue-dependent inflammatory signals that use the IL-17RA subunit, including IL-17F, IL-17C, and IL-25 and nonIL-17 cytokines such as IL-1 family members. Since Regnase-1 acts on T cells and macrophages, it is also surprising that our BM chimera and RTEC-specific deletion studies indicated that the impact of Regnase-1 in AGN seemed to be so RTEC specific $(35,56)$. However, this finding does suggest that a major function of Regnase-1 is to restrain downstream IL-17R-mediated signals in the kidney. We previously reported that mice with a Regnase-1 deficiency have increased resistance to kidney infections caused by systemic Candida albicans infections (33). These data indicate that, while constraint of IL-17 signaling by Regnase- 1 is beneficial in preventing autoimmunity, this same inhibition limits the efficacy of IL-17 in mediating renal host defense; thus, care will be needed when considering targeting this pathway pharmacologically. Although Cre-lox technology has enabled manipulation of gene expression in targeted tissues, off-target effects of Cre sometimes occur. However, Cdh16Cre mice are a well-characterized and extensively used mouse system with no reported off-target activity.

This work highlights an inflammatory signaling axis mediated by IL-17 and Regnase- 1 and the transcription factor $\mathrm{I} \kappa \mathrm{B} \zeta$. The gene encoding $\mathrm{I} \kappa \mathrm{B} \zeta(N f k b i z)$ is transcriptionally induced by IL-17, thus acting as a feed-forward activator of IL-17 signaling $(57,58)$. I $\mathrm{B} \zeta$ $\zeta$ regulates transcription of $L c n 2$ and $I l 6(58)$. Lcn2 is a well-known biomarker of kidney disease (27) and causes renal damage by inducing apoptosis of RTECs $(26,33,59)$. This observation likely explains why we observed such a potent impact of negative 
A
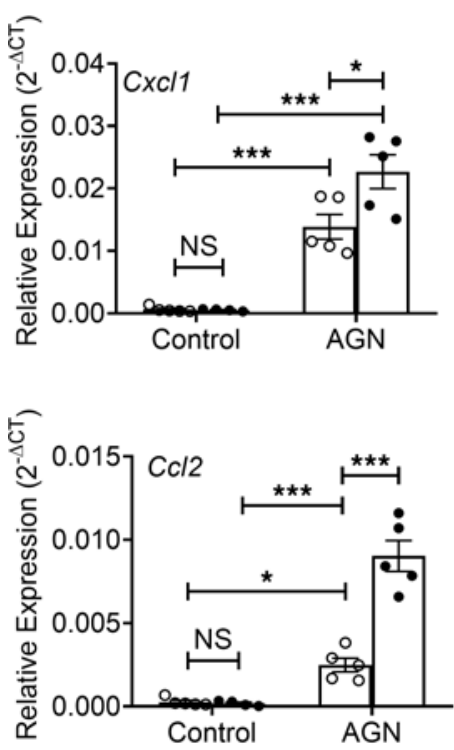

B

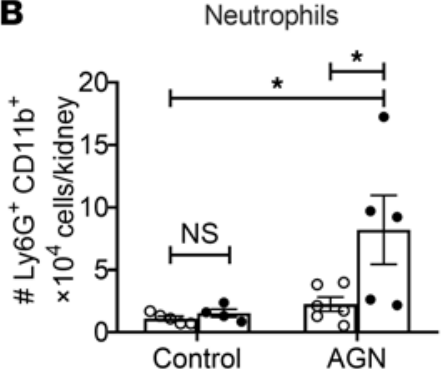

C

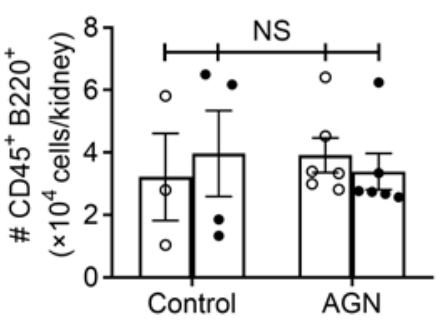

D

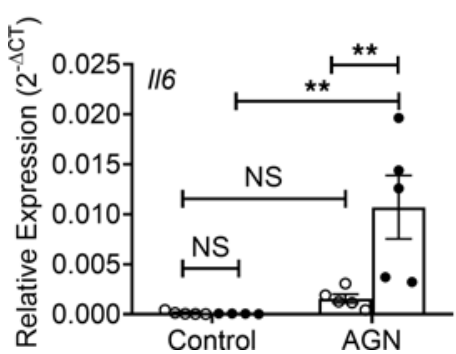

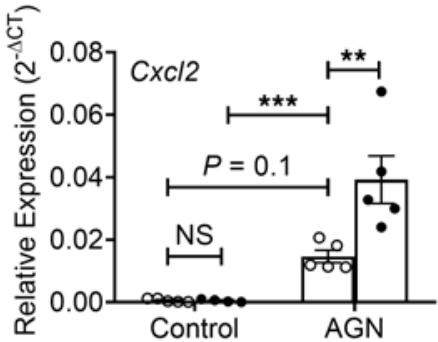

- Zc3h12a $a^{f / f t}$

- Zc3h12a $a^{\text {Cdh16 }}$
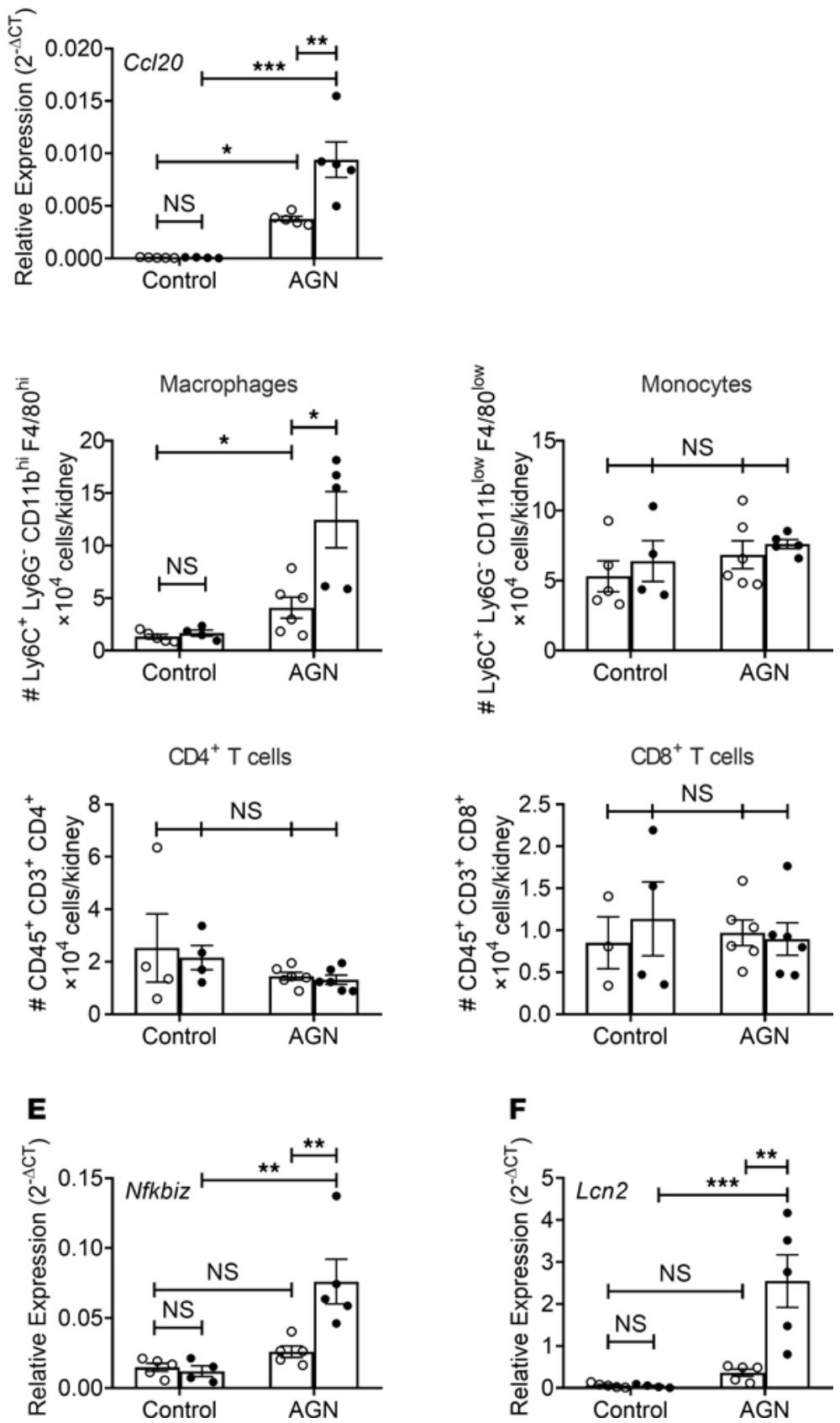

Figure 4. Increased inflammatory gene expression and cell infiltration in the nephritic kidney of RTEC-specific Regnase-1 deficient mice. Zc $3 h 12 a^{f / f l}$ and Zc3h12a cdh16 mice ( $n=3-6)$ were subjected to AGN. (A-F) At day 7 p.i., renal Cxcl1, Cxcl2, Ccl20 and Ccl2 mRNA (A) expression was measured by qPCR; neutrophil, macrophage, monocyte, B cell, CD4+ T cell, and CD8+ T cell infiltration in the kidney was quantified by flow cytometry (B and C); and II6 (D), Nfkbiz (E), and $\operatorname{Lcn} 2$ (F) transcript expression was evaluated by qPCR. Expression was normalized to Gapdh (A, D, E, and F). The data is pooled from at least 2 independent experiments. Statistical analysis by 2-way ANOVA. ${ }^{*} P<0.05 ;{ }^{* *} P<0.01 ;{ }^{* * *} P<0.001$. 
regulatory mechanisms of IL-17 signaling in the development of nephritic pathology. We also observed that IL-17 synergizes with TNF- $\alpha$ in inducing NFKBIZ, IL6, and LCN2 in HK-2 cell lines lacking Regnase-1 (ZC3H12A). TNF- $\alpha$ on its own does not activate NFKBIZ (60); therefore, this result reveals a mechanism by which IL-17 and TNF- $\alpha$ together drive the expression of $\mathrm{I} \kappa \mathrm{B} \zeta$ and its responsive genes.

Understanding how IL-17 and its downstream signals are regulated is important from a basic science perspective and has potential translational applications. Currently, approximately 90 clinical trials are ongoing to test IL-17 blockade (clinicaltrials.gov; NCT01107457, NCT00966875, NCT01539213, NCT00936585, and NCT03073213). We previously showed that blocking IL-17 alleviates symptoms in mouse models of AGN (10). Antibodies against IL-23, the cytokine responsible for generating pathogenic type 17 cells, are showing promising results in clinical trials in number of inflammatory diseases (clinicaltrials.gov: accession nos. NCT01845987, NCT01947933, and NCT04630652). Hence, future studies will need to focus on conducting preclinical trials to assess the efficacy of anti-IL-23, anti-IL-6, and anti-IL-1 therapies, the upstream regulators of type 17 cells development, in mouse model of AGN. Here, we have identified a potentially novel role for the endoribonuclease Regnase-1

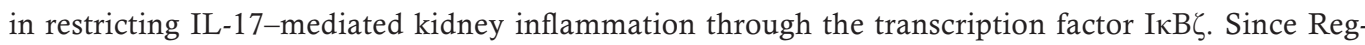
nase-1 negatively regulates pathogenic IL-17 activity in the nephritic kidney, these results may ultimately have the potential to provide new targets for therapies against AGN.

\section{Methods}

Mice. C57BL/6NTac (WT) mice were from Taconic Biosciences Inc. $1 l 17 \mathrm{ra}^{-1-}$ mice were from Amgen and bred in-house. Il17 $\mathrm{ra}^{f l f l}$ mice were provided by Jay Kolls (Tulane University). Zc3h12aflfl mice are under material transfer agreement (MTA) from University of Central Florida (Orlando, Florida, USA). Cdh16 ${ }^{\text {Cre }}$ (Ksp1.3 ${ }^{\text {Cre }}$ ) mice and B6.SJL-Ptprc (B6 CD45.1) were from The Jackson Laboratory. All the experiments used age-matched controls of both sexes, housed in specific pathogen-free conditions.

$A G N$. Mice are immunized i.p. with $0.2 \mathrm{mg}$ of rabbit IgG (011-000-003, Jackson ImmunoResearch) in CFA (MilliporeSigma). Controls received CFA only. Three days later, mice were injected i.v. with heat-inactivated rabbit anti-mouse GBM serum (Custom-made, Lampire Biological Lab Inc.) at 5 $\mathrm{mg} / 20 \mathrm{~g}$ body weight. Mice were sacrificed at day 7 or 14 after anti-GBM injection. Serum blood urea nitrogen (BUN) was measured using Blood Urea Nitrogen Enzymatic kit (Bioo Scientific Corp.) and creatinine with QuantiChrom Creatinine Assay kit (BioAssay Systems).

To create BM chimeras, mice were sublethally irradiated (9 Grey) and, 24 hours later, $5 \times 10^{6}$ to 10 $\times 10^{6}$ donor BM cells (either CD45.1 or CD45.2) were injected i.v. After 6-8 weeks, peripheral blood of recipients was tested for reconstitution with donor BM cells by flow cytometry for CD45.1 and CD45.2.

Deletion of ZC3H12A in HK-2 cells. KO of ZC3H12A in the HK-2 cell line was performed by SYNTHEGO Company using CRISPR-Cas9. Briefly, HK-2 cells were electroporated with Cas9 and a ZC3H12A-specific sgRNA (5'-GACACAUACCGUGACCUCCA-3'). Isogenic negative control HK-2 cells were electroporated with only Cas9. Editing efficiency was evaluated 48 hours after transfection. The cells were expanded to get a KO cell pool. Clonal ZC3H12A-KO cell lines were generated by limiting dilution. Genomic DNA from monoclonal populations were verified by PCR and Sanger sequencing using $Z C 3 H 12 A$ specific primer (forward, 5'-TGACCTTGGCGTTAACCACTC -3'; reverse, 5'-GTGGACCCCAAGTCTGTCAG -3'). The sequencing results were analyzed using the Inference of CRISPR Edits (ICE) tool (https://ice.synthego.com/\#/) and NCBI nucleotide blast tool.

Immunoblot analysis. HK-2 cells (ATCC) were lysed in 1× NP-40 lysis buffer supplemented with protease inhibitor cocktail. Lysates were separated by SDS-PAGE, transferred to polyvinylidene difluoride membranes, and imaged with the enhanced chemiluminescence detection system (Thermo Fisher Scientific) and developed with a FluorChem E imager (ProteinSimple). Immunoblotting antibodies: human/mouse anti-Regnase-1 (MAB7875, R\&D Systems), human anti- $\beta$ actin (ab49900, Abcam), human anti-C/EBP $\delta$ (sc-365546, Santa Cruz Biotechnology Inc.), human/mouse anti-C/

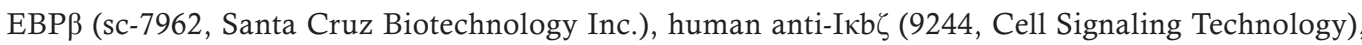
and human/mouse anti-YY1 (sc-7341, Santa Cruz Biotechnology Inc.). The intensity of the protein band was measured by ImageJ software (NIH).

ELISA. HK-2 cells (ATCC) were stimulated with IL-17 and/or TNF- $\alpha$ for different times. The supernatant was collected, and ELISA was performed to detect IL-6 ( 8 hours after stimulation, catalog 88-7066-22, Invitrogen) and LCN2 (24 hours after stimulation, catalog EHLCN2, Invitrogen). 
A ZC3H12A: EXON 4
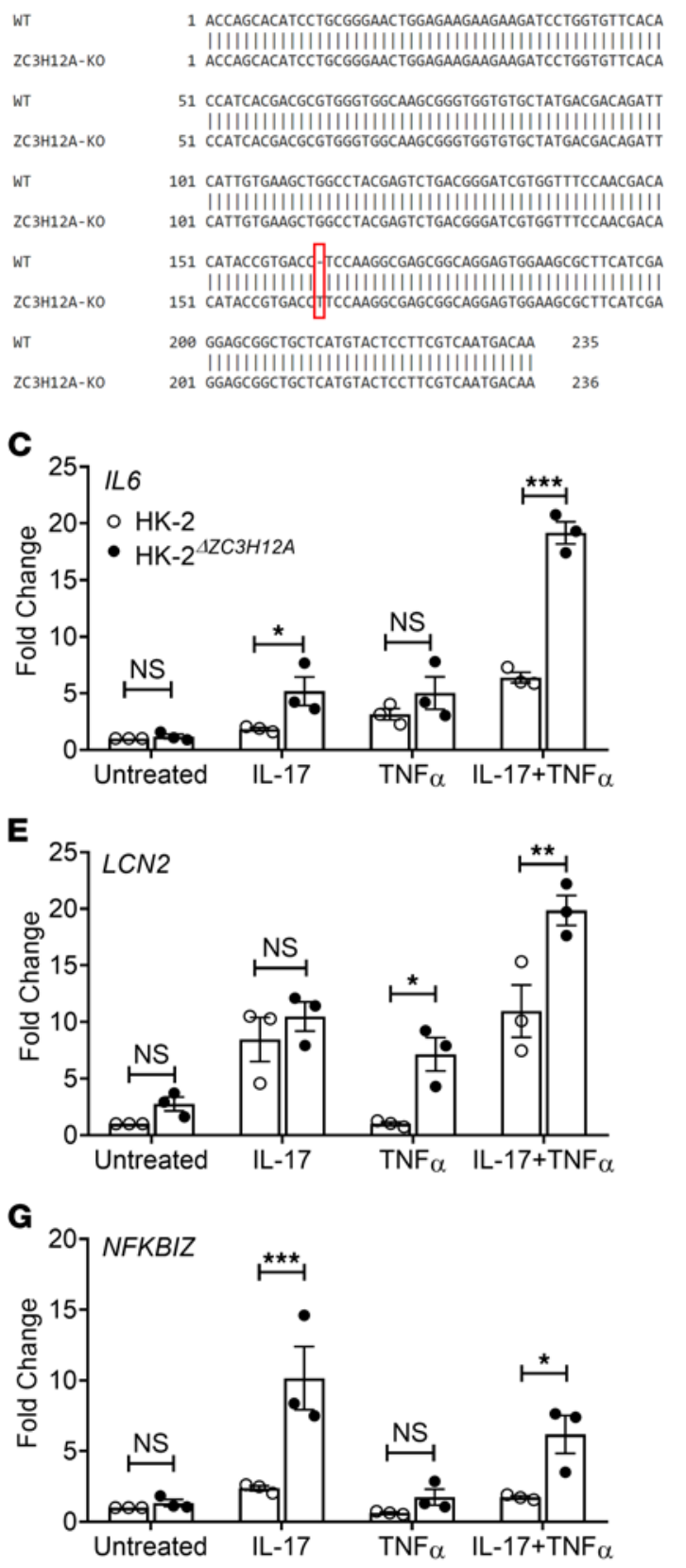

B

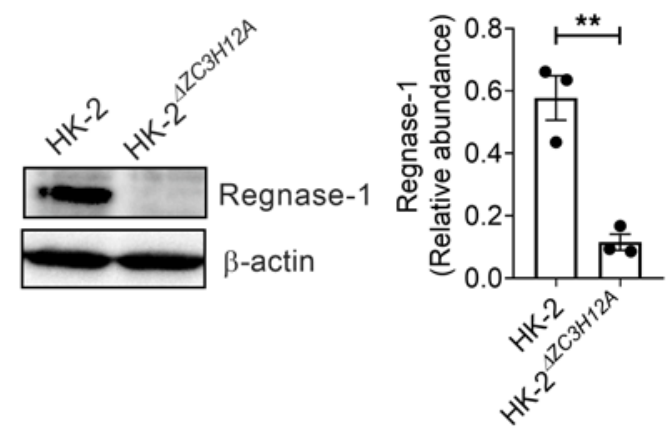

D

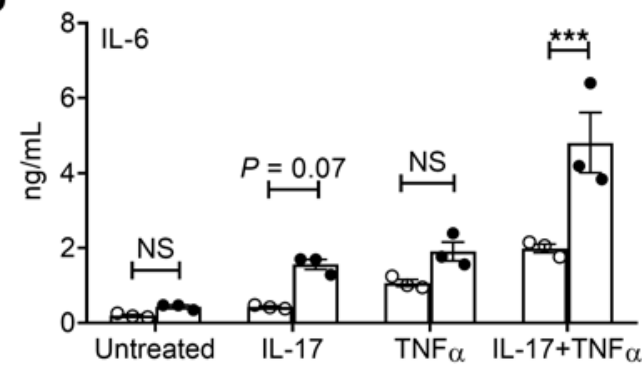

$\mathbf{F}$
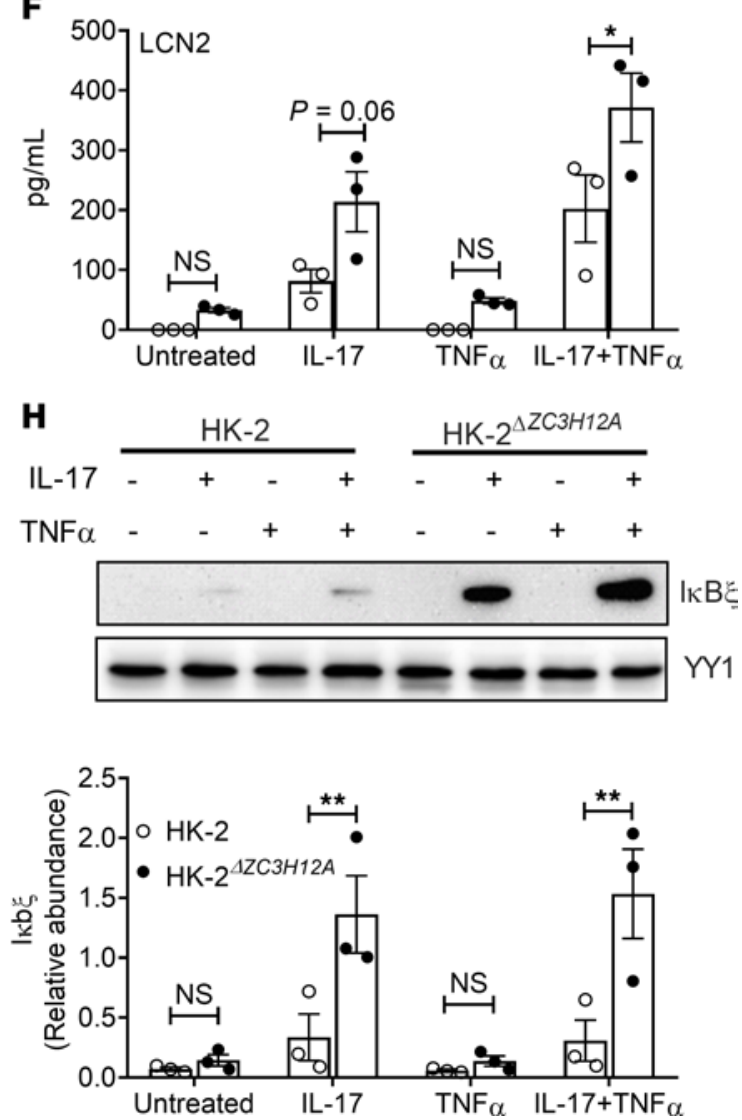

Figure 5. Deletion of ZC3H12A gene elevates inflammatory mediator expression in the HK-2 cell line. (A) Experimental strategy to delete $Z C 3 H 12 A$ gene by CRISPR-Cas9 in the HK-2 cell line. (B) HK-2 and HK-2 ${ }^{4 Z 23 H 2 A}$ cells were lysed and evaluated for Regnase- 1 by Western blot. Protein relative abundance and representative image are shown. (C, E, and G) HK-2 and HK-2 ${ }^{4 Z C 3 H 12 A}$ cells were stimulated with IL-17 and/or TNF- $\alpha$ and mRNA expression of IL6, LCN2, and NFKBIZ was measured by qPCR (8 hours after stimulation), normalized to GAPDH. (D and F) Protein level of IL-6 (8 hours after stimulation) and LCN2 (24 hours after stimulation) in culture supernatant were measured by ELISA. (H) HK-2 and HK-2 ${ }^{42 C 3 H 12 A}$ cells were stimulated with IL-17 and/or TNF- $\alpha$ for 8 hours, and cell lysates were evaluated for $1 \kappa B \xi$ by Western blot. Protein relative abundance and representative image are shown. Data pooled from at least 3 independent experiments. Statistical analysis by 2-tailed unpaired $t$ test (B) or 2-way ANOVA (C-H). ${ }^{*} P<0.05 ;{ }^{* *} P<0.01 ;{ }^{* *} P<0.001$. 
A

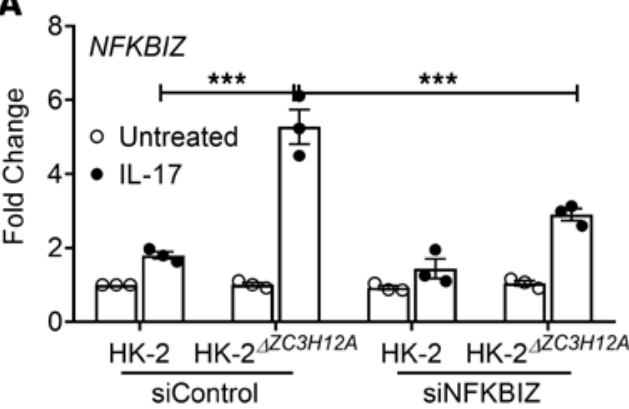

B
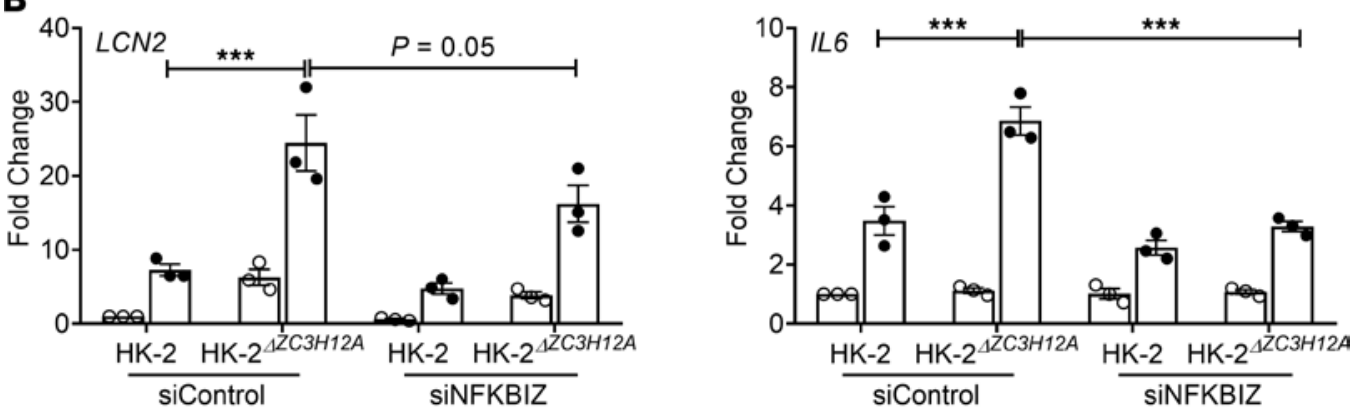

C
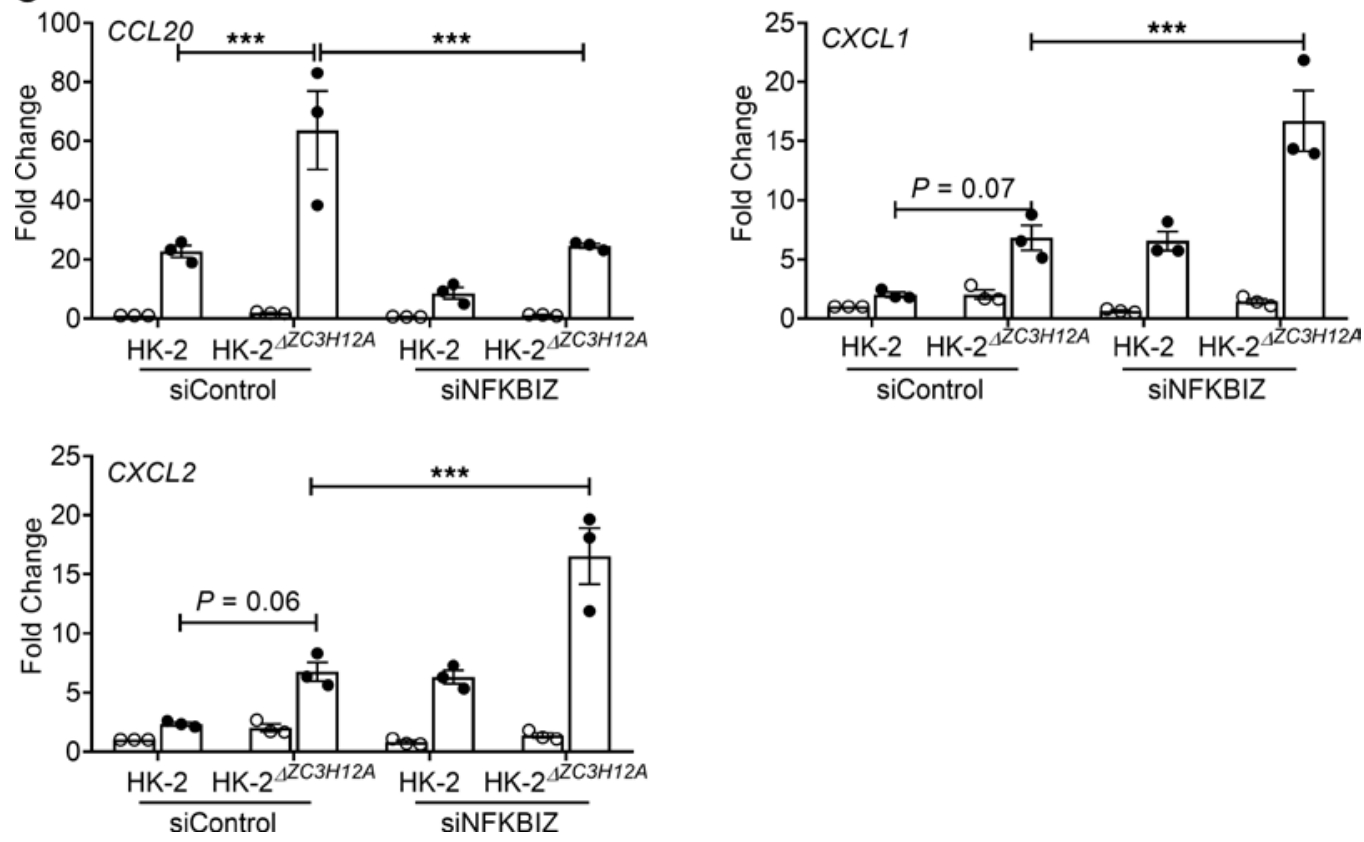

Figure 6. Regnase-1 upregulates IL6 and LCN2 gene expression via NFKBIZ. HK-2 and HK-2 ${ }^{4 Z C 3 H 12 A}$ cells were either treated with siRNA against NFKBIZ or control siRNA. (A) Efficiency of NFKBIZ gene silencing was measured by qPCR. (B and C) Cells were either stimulated with IL-17 or left untreated for 8 hours and analyzed for IL6, LCN2, CCL20, CXCL1, and $C X C L 2$ gene expression by qPCR. Data pooled from at least 3 independent experiments. Statistical analysis by 2-way ANOVA. ${ }^{* * *} P<0.001$.

Luciferase assay. Luciferase assay was performed as previously described (61). Briefly, IL-17 Reporter HEK 293 cells were seeded in 12-well plates. In total, $100 \mathrm{ng}$ of LCN2 promoter luciferase plasmid and 5 ng of Renilla luciferase plasmid were cotransfected. Cells were stimulated with $10 \mathrm{ng} / \mathrm{mL}$ of human IL-17 for 14 hours and then lysed for luciferase analyze by using GloMax Navigator Microplate Luminometer.

Histology and flow cytometry. For IHC, kidneys were fixed with $10 \%$ buffered formaldehyde and embedded in paraffin. Slices of $4 \mu \mathrm{m}$ thick were stained with H\&E or periodic acid-Schiff (PAS) and observed on an EVOS microscope (Thermo Fisher Scientific). The slides were scored blindly by an expert with 
more than 10 years of experience with renal histopathology, as described previously (62). Briefly, severity of GN was assessed by mild to moderate increase in mesangial cellularity, thickening of the GBM, endocapillary hypercellularity, and crescents formation. The tubulointerstitial inflammation was measured by assessing tubular atrophy and tubulointerstitial inflammation.

Kidneys were perfused with PBS containing EDTA (MilliporeSigma) before harvesting. Kidneys were digested at $37^{\circ} \mathrm{C}$ in $1 \mathrm{mg} / \mathrm{mL}$ collagenase IV (Worthington) in complete RPMI for 30 minutes, filtered through $70 \mathrm{~mm}$ strainers, and washed twice in PBS. For flow cytometry, the following antibodies were used: CD45 (30-F11, eBioscience), CD3 (145-2C11, BioLegend), CD4 (GK1.5, BioLegend), CD8 (53-6.7, BioLegend), CD45R/B220 (RA3-6B2, BioLegend), Ly6G (IA8, eBioscience), CD11b (M1/70, BioLegend), F4/80 (BM8, BioLegend), and Ly6C (HK1.4, eBioscience). Samples were acquired on BD LSRFortessa cytometer (BD Biosciences) and analyzed by FlowJo software (Tree Star Inc.).

$R N A$ silencing and $q P C R$. ON-TARGETplus SMARTpool siRNAs targeting human NFKBIZ were from Dharmacon. For RNA silencing, HK-2 cells were seeded overnight in antibiotic-free DMEM/ F12 media (Thermo Fisher Scientific) with supplements. Transfection was performed 18 hours later with $50 \mathrm{nM}$ siRNAs with DharmaFECT Reagent 1. Culture media was replaced after 24 hours. One day later, cells were treated with human IL-17 and human TNF- $\alpha$ (Peprotech) for 8 hours.

RNA was extracted using RNeasy kits (Qiagen). Complementary DNA was synthesized by SuperScript III First Strand Kits (Thermo Fisher Scientific). Quantitative PCR (qPCR) was performed with the PerfeCTa SYBR Green FastMix (Quanta BioSciences) and analyzed on an ABI 7300 real-time instrument. Primers were from QuantiTect Primer Assays (Qiagen). Expression was normalized to mouse or human Gapdh.

Statistics. All data are shown as Mean \pm SEM. Statistical analyses were performed using 1- or 2-tailed Student's $t$ test and 1- or 2-way ANOVA depending on the experiment using GraphPad Prism. A $P$ value less than 0.05 was considered as statistically significant. ${ }^{*} P<0.05 ;{ }^{*} P<0.01 ;{ }^{* *} P<0.001$. All experiments were performed at least twice in independent replicates.

Study approval. All the experiments were conducted following NIH guidelines under protocols approved by the University of Pittsburgh IACUC (protocol no. 20087922).

\section{Author contributions}

DDL, JKK, SLG, and PSB designed the experiments; DDL, RB, KR, CVJ, YL, and PSB performed the experiments. JKK provided conditional KO mouse. DDL, SLG, and PSB analyzed and interpreted the data and wrote the manuscript.

\section{Acknowledgments}

This work was supported by a Rheumatology Research Foundation grant to SLG and PSB and NIH grants AI145242 (SLG and PSB), AI147383 (SLG), DK104680 (PSB), AI142354 (PSB), and R35 HL139930 (JKK). We thank P.K. Kollatukudy, M.J. McGeachy, K. Chen, S. Majumder, and G. Trevejo-Nunez for valuable input and B. Coleman and L. Lin for assistance with mouse breeding.

Address correspondence to: Partha S Biswas or Sarah L Gaffen, S725 BST, Department of Medicine, University of Pittsburgh, 200 Lothrop Street, Pittsburgh, Pennsylvania 15261, USA. Phone: 412.648.8708; Email: psb13@pitt.edu. (PSB); Phone: 412.383.8903; Email: sarah.gaffen@pitt.edu.

1. McAdoo SP, Pusey CD. Anti-glomerular basement membrane disease. Clin J Am Soc Nephrol. 2017;12(7):1162-1172.

2. Segelmark M, Hellmark T. Autoimmune kidney diseases. Autoimmun Rev. 2010;9(5):A366-A371.

3. Moroni G, Ponticelli C. Rapidly progressive crescentic glomerulonephritis: early treatment is a must. Autoimmun Rev. 2014;13(7):723-729

4. Webster P, Pusey C. Glomerular disease: crescentic glomerulonephritis: beyond the immune system. Nat Rev Nephrol. 2017;13(4):198-200

5. Waldman M, Appel GB. Update on the treatment of lupus nephritis. Kidney Int. 2006;70(8):1403-1412.

6. Davidson A. What is damaging the kidney in lupus nephritis? Nat Rev Rheumatol. 2016;12(3):143-153.

7. Hodgkins KS, Schnaper HW. Tubulointerstitial injury and the progression of chronic kidney disease. Pediatr Nephrol. 2012;27(6):901-909

8. Qi R, Yang C. Renal tubular epithelial cells: the neglected mediator of tubulointerstitial fibrosis after injury. Cell Death Dis. 2018;9(11):1126.

9. Shlomchik MJ, et al. From T to B and back again: positive feedback in systemic autoimmune disease. Nat Rev Immunol. 
2001;1(2):147-153.

10. Ramani K, et al. An essential role of interleukin-17 receptor signaling in the development of autoimmune glomerulonephritis J Leukoc Biol. 2014;96(3):463-472.

11. Turner JE, et al. The Th17 immune response in renal inflammation. Kidney Int. 2010;77(12):1070-1075.

12. Turner JE, et al. IL-17A production by renal $\gamma \delta$ T cells promotes kidney injury in crescentic GN. $J$ Am Soc Nephrol. 2012;23(9):1486-1495.

13. Paust HJ, et al. The IL-23/Th17 axis contributes to renal injury in experimental glomerulonephritis. J Am Soc Nephrol. 2009;20(5):969-979.

14. Pisitkun $\mathrm{P}$, et al. The adaptor protein CIKS/ACT1 is necessary for collagen-induced arthritis, and it contributes to the production of collagen-specific antibody. Arthritis Rheum. 2010;62(11):3334-3344.

15. Schmidt T, et al. Mechanisms and functions of IL-17 signaling in renal autoimmune diseases. Mol Immunol. 2018;104:90-99.

16. St Leger AJ, et al. STAT-3-independent production of IL-17 by mouse innate-like alphabeta T cells controls ocular infection. J Exp Med. 2018;215(4):1079-1090.

17. Mitsdoerffer M, et al. Proinflammatory T helper type 17 cells are effective B-cell helpers. Proc Natl Acad Sci U S A. 2010;107(32):14292-14297.

18. Bartlett HS, Million RP. Targeting the IL-17-T(H)17 pathway. Nat Rev Drug Discov. 2015;14(1):11-12.

19. Patel DD, et al. Effect of IL-17A blockade with secukinumab in autoimmune diseases. Ann Rheum Dis. 2013;72 Suppl 2:ii116-ii123.

20. Krebs CF, et al. Pathogen-induced tissue-resident memory $\mathrm{T}_{\mathrm{H}} 17\left(\mathrm{~T}_{\mathrm{RM}} 17\right)$ cells amplify autoimmune kidney disease. Sci Immunol. 2020;5(50):eaba4163.

21. Ooi JD, et al. IL-23, not IL-12, directs autoimmunity to the Goodpasture antigen. J Am Soc Nephrol. 2009;20(5):980-989.

22. Zhang Z, et al. The role of IL-23/IL-17 axis in lupus nephritis. J Immunol. 2009;183(5):3160-3169.

23. Koga T, et al. T cells and IL-17 in lupus nephritis. Clin Immunol. 2017;185:95-99.

24. Ramani K, Biswas PS. Emerging roles of the Th17/IL-17-axis in glomerulonephritis. Cytokine. 2016;77:238-244.

25. Sonder SU, et al. IL-17-induced NF-kappaB activation via CIKS/Act1: physiologic significance and signaling mechanisms. J Biol Chem. 2011;286(15):12881-12890.

26. Viau A, et al. Lipocalin 2 is essential for chronic kidney disease progression in mice and humans. J Clin Invest. 2010;120(11):4065-4076.

27. Torres-Salido MT, et al. Neutrophil gelatinase-associated lipocalin as a biomarker for lupus nephritis. Nephrol Dial Transplant 2014;29(9):1740-1749.

28. Haase M, et al. Neutrophil gelatinase-associated lipocalin: a superior biomarker for detection of subclinical acute kidney injury and poor prognosis. Biomark Med. 2011;5(4):415-417.

29. Qian Y, et al. The adaptor Act1 is required for interleukin 17-dependent signaling associated with autoimmune and inflammatory disease. Nat Immunol. 2007;8(3):247-256.

30. Chang SH, et al. Act1 adaptor protein is an immediate and essential signaling component of interleukin-17 receptor. $J$ Biol Chem. 2006;281(47):35603-35607.

31. Anderson P. Post-transcriptional regulons coordinate the initiation and resolution of inflammation. Nat Rev Immunol. 2010;10(1):24-35.

32. O'Connell RM, et al. MicroRNA-155 promotes autoimmune inflammation by enhancing inflammatory T cell development. Immunity. 2010;33(4):607-619.

33. Garg AV, et al. MCPIP1 endoribonuclease activity negatively regulates interleukin-17-mediated signaling and inflammation. Immunity. 2015;43(3):475-487.

34. Mao R, et al. Regnase-1, a rapid response ribonuclease regulating inflammation and stress responses. Cell Mol Immunol. 2017;14(5):412-422.

35. Jeltsch KM, et al. Cleavage of roquin and regnase-1 by the paracaspase MALT1 releases their cooperatively repressed targets to promote T(H)17 differentiation. Nat Immunol. 2014;15(11):1079-1089.

36. Du Y, et al. Experimental anti-GBM nephritis as an analytical tool for studying spontaneous lupus nephritis. Arch Immunol Ther $\operatorname{Exp}$ (Warsz). 2008;56(1):31-40.

37. Shao X, et al. Epithelial-specific Cre/lox recombination in the developing kidney and genitourinary tract. J Am Soc Nephrol. 2002;13(7):1837-1846

38. Ramani $\mathrm{K}$, et al. Unexpected kidney-restricted role for IL-17 receptor signaling in defense against systemic Candida albicans infection. JCI Insight. 2018;3(9):e98241.

39. Liang J, et al. MCP-induced protein 1 deubiquitinates TRAF proteins and negatively regulates JNK and NF-kappaB signaling JExp Med. 2010;207(13):2959-2973.

40. Miao R, et al. Targeted disruption of MCPIP1/Zc3h12a results in fatal inflammatory disease. Immunol Cell Biol. 2013;91(5):368-376

41. Monin L, et al. MCPIP1/Regnase-1 restricts IL-17A- and IL-17C-dependent skin inflammation. J Immunol. 2017;198(2):767-775.

42. Ruiz-Romeu E, et al. MCPIP1 RNase is aberrantly distributed in psoriatic epidermis and rapidly induced by IL-17A. J Invest Dermatol. 2016;136(8):1599-1607.

43. Han WK, et al. Kidney injury molecule-1 (KIM-1): a novel biomarker for human renal proximal tubule injury. Kidney Int. 2002;62(1):237-244

44. Karlsen JR, et al. Induction of neutrophil gelatinase-associated lipocalin expression by co-stimulation with interleukin-17 and tumor necrosis factor-alpha is controlled by IkappaB-zeta but neither by C/EBP-beta nor C/EBP-delta. J Biol Chem 2010;285(19):14088-14100.

45. Ruddy MJ, et al. Functional cooperation between interleukin-17 and tumor necrosis factor-alpha is mediated by CCAAT/ enhancer-binding protein family members. J Biol Chem. 2004;279(4):2559-2567.

46. Kurts C, et al. The immune system and kidney disease: basic concepts and clinical implications. Nat Rev Immunol. 2013;13(10):738-753.

47. Korn T, et al. IL-17 and Th17 Cells. Annu Rev Immunol. 2009;27:485-517.

48. Targan SR, et al. A randomized, double-blind, placebo-controlled phase 2 study of brodalumab in patients with moderate-to-severe 
crohn's disease. Am J Gastroenterol. 2016;111(11):1599-1607.

49. Lee JS, et al. Interleukin-23-independent IL-17 production regulates intestinal epithelial permeability. Immunity. 2015;43(4):727-738

50. Maxwell JR, et al. Differential roles for interleukin-23 and interleukin-17 in intestinal immunoregulation. Immunity. 2015;43(4):739-750

51. McGeachy MJ, et al. The IL-17 family of cytokines in health and disease. Immunity. 2019;50(4):892-906.

52. Riedel JH, et al. IL-17F promotes tissue injury in autoimmune kidney diseases. J Am Soc Nephrol. 2016;27(12):3666-3677.

53. Krohn S, et al. IL-17C/IL-17 receptor E signaling in CD4 ${ }^{+} \mathrm{T}$ cells promotes $\mathrm{T}_{\mathrm{H}} 17$ cell-driven glomerular inflammation. $J$ Am Soc Nephrol. 2018;29(4):1210-1222.

54. Bar E, et al. IL-17 regulates systemic fungal immunity by controlling the functional competence of NK cells. Immunity. 2014;40(1):117-127

55. Liu F, et al. Distinct fate, dynamics and niches of renal macrophages of bone marrow or embryonic origins. Nat Commun. 2020;11(1):2280.

56. Akira S. Regnase-1, a ribonuclease involved in the regulation of immune responses. Cold Spring Harb Symp Quant Biol. 2013;78:51-60.

57. Annemann M, et al. Atypical IкB proteins in immune cell differentiation and function. Immunol Lett. 2016;171:26-35.

58. Sun SC. The non-canonical NF-кB pathway in immunity and inflammation. Nat Rev Immunol. 2017;17(9):545-558.

59. Onishi RM, Gaffen SL. Interleukin-17 and its target genes: mechanisms of interleukin-17 function in disease. Immunology. 2010;129(3):311-321.

60. Willems M, et al. IкB $\zeta$ : an emerging player in cancer. Oncotarget. 2016;7(40):66310-66322.

61. Shen $\mathrm{F}$, et al. Identification of common transcriptional regulatory elements in interleukin-17 target genes. $J$ Biol Chem. 2006;281(34):24138-24148.

62. Li QZ, et al. The lupus-susceptibility gene kallikrein downmodulates antibody-mediated glomerulonephritis. Genes Immun. 2009;10(5):503-508 